\title{
7 Zusammenfassung und Schlussfolgerungen
}

Mit der vorliegenden Studie wurden die Handlungen, die Pflegende in der neurologischen (Früh-)Rehabilitation durchführen, dargestellt. Ebenso wurden die komplexen Zusammenhänge zur Durchführung dieser beschrieben und eine Theorie. zur therapeutischen Pflege für den Fachbereich der neurologischen (Früh-) Rehabilitation entwickelt. Die Ergebnisse dieser Arbeit zeigen, was therapeutische Pflege beinhaltet, und in welchen Bereichen Bildung für Pflegende im Fachbereich der neurologischen (Früh-)Rehabilitation notwendig ist, um therapeutisch pflegen zu können. Nicht zuletzt geht diese Forderung aus der OPS-Ziffer 8-552 hervor, in der die Durchführung durch besonders geschultes Personal gefordert wird. Gleichzeitig kann daraufhin kritisch betrachtet werden, wie viele Qualifizierungsmöglichkeiten in diesem Fachbereich angeboten werden, wie viel Pflegepersonal außer der Ausbildung über Weiterqualifizierungen in diesem Fachbereich verfügt, und inwiefern Ergebnisse dieser Arbeit bereits in Aus-Fort- und Weiterbildungen berücksichtigt werden. Weiterhin ist zu fragen, wie viele Stunden Weiterbildung ausreichend sind, um für die Anforderungen und zukünftigen Herausforderungen in diesem Bereich ausgestattet zu werden, und um als besonders geschult im Hinblick auf die therapeutische Pflege zu gelten. Dieser Aspekt gewinnt besondere Relevanz vor dem Hintergrund der demografischen aber auch epidemiologischen Entwicklung mit dem Anstieg der Inzidenz von Schlaganfällen, Schädelhirntraum (SHT) und chronischen Erkrankungen, um nur einige zu nennen. Pflegende können in Zusammenarbeit mit dem therapeutischen Team einen entscheidenden Beitrag zur Rehabilitation betroffener Menschen in der neurologischen (Früh-) Rehabilitation leisten, oder um es in den Worten von Virginia Henderson auszudrücken, die sich schon zu Beginn der 80er Jahre wünschte: ,that nurses will become rehabilitators par excellence ..." (Henderson, 1980, S. 246). Jedoch kann dieser Wunsch nach Myco (1984) erst realisiert werden: ,, Until nurses develop the confidence, through acquisition of appropriate knowledge and skill ...” (Myco, 1984, S. 436). 